\title{
The trans-Planckian problem as a guiding principle
}

\author{
L.C. Barbado, ${ }^{a}$ C. Barceló, ${ }^{a}$ L.J. Garay ${ }^{b, c}$ and G. Jannes ${ }^{d}$ \\ ${ }^{a}$ Instituto de Astrofísica de Andalucía (IAA - CSIC), \\ Glorieta de la Astronomía, 18008 Granada, Spain \\ ${ }^{b}$ Departamento de Física Teórica II, Universidad Complutense de Madrid, \\ 28040 Madrid, Spain \\ ${ }^{c}$ Instituto de Estructura de la Materia (IEM - CSIC), \\ Serrano 121, 28006 Madrid, Spain \\ ${ }^{d}$ Low Temperature Laboratory, Aalto University School of Science, \\ P.O. Box 15100, 00076 Aalto, Finland \\ E-mail: luiscb@iaa.es, carlos@iaa.es, luisj.garay@fis.ucm.es, \\ jannes@ltl.tkk.fi
}

ABSTRACT: We use the avoidance of the trans-Planckian problem of Hawking radiation as a guiding principle in searching for a compelling scenario for the evaporation of black holes or black-hole-like objects. We argue that there exist only three possible scenarios, depending on whether the classical notion of long-lived horizon is preserved by high-energy physics and on whether the dark and compact astrophysical objects that we observe have longlived horizons in the first place. Along the way, we find that i) a theory with high-energy superluminal signalling and a long-lived trapping horizon would be extremely unstable in astrophysical terms and that ii) stellar pulsations of objects hovering right outside but extremely close to their gravitational radius can result in a mechanism for Hawkinglike emission.

KEYwords: Black Holes, Models of Quantum Gravity

ARXIV EPRINT: 1109.3593 


\section{Contents}

1 Introduction 1

2 Preliminaries 3

3 Subluminal dispersion $\quad 4$

4 Superluminal dispersion $\quad 5$

5 Avoidance of long-lasting horizons $\quad 7$

6 Summary and conclusions $\quad 12$

$\begin{array}{ll}\text { A Stability analysis } & 13\end{array}$

\section{Introduction}

A remarkable characteristic of the general-relativistic theoretical framework, encompassing classical as well as semiclassical aspects, is that it seems to contain the seeds of its own destruction. We shall argue that, when properly interpreted, this framework also incorporates clues for efficiently debugging its problems. For instance, classical general relativity predicts the unavoidable formation of black holes through gravitational collapse. But then, the classical laws of black hole dynamics call openly for quantum mechanics for consistency. A crucial step towards this consistency was Hawking's derivation that black holes should emit thermal radiation, and therefore evaporate, due to quantum effects $[1,2]$. However, right after Hawking presented his derivation, Unruh showed that it was somewhat untrustable due to what has come to be known as the trans-Planckian problem [3]. Hawking radiation appears to come from the red-shifting by the collapsing geometry of modes with huge initial frequencies, well beyond the Planck frequency $\omega_{\mathrm{P}}$. To estimate how huge these frequencies are, one only has to calculate the blue-shift factor $e^{\kappa_{\mathrm{H}} t_{\mathrm{BH}}}$, where $\kappa_{\mathrm{H}}$ stands for the surface gravity of the initial black hole $\left(\kappa_{\mathrm{H}}=(4 m)^{-1}\right.$, with $m$ its mass) and $t_{\mathrm{BH}}$ is the lifetime of the evaporating black hole $\left(t_{\mathrm{BH}}=5120 \pi\left(\mathrm{m} / m_{\mathrm{P}}\right)^{3} t_{\mathrm{P}}\right.$, where $m_{\mathrm{P}}$ and $t_{\mathrm{P}}$ are the Planck mass and time respectively), and multiply it by the typical frequencies of Hawking radiation, of the order of $\kappa_{\mathrm{H}}$ (for the argument that follows, it is not necessary to take into account the variation of $\kappa_{\mathrm{H}}$ during the evaporation). For a Solar mass black hole $\left(\kappa_{\mathrm{H}} \simeq 10^{-39} \omega_{\mathrm{P}}\right.$ and $\left.t_{\mathrm{BH}} \sim 10^{118} t_{\mathrm{P}}\right)$ this calculation yields a value

$$
\omega_{\max } \sim \kappa_{\mathrm{H}} e^{\kappa_{\mathrm{H}} t_{\mathrm{BH}}} \sim 10^{10^{79}} \omega_{\mathrm{P}}
$$

for the highest invoked frequency $\omega_{\max }$. Just one second after the ignition of the black hole [4] the highest frequencies involved in the Hawking process are already of the order 
$\omega_{\max } \sim 10^{100000} \omega_{\mathrm{P}}$. It is extremely difficult to believe in the validity of quantum field theory in a curved classical background up to these enormous energy scales. As Unruh used to say, this should be considered the trans-Planckian disaster or, perhaps, the trans-Planckian catastrophe, in analogy with the ultraviolet catastrophe of the late 19th century. In other words, the evaporating black hole scenario based on this mechanism for Hawking radiation can be considered strongly unsatisfactory.

At these extremely large energy scales the geometry itself should be "quantum" (using the word in a vague sense) and modify the effective behaviour of the matter fields. If one could prove that Hawking emission is not really dependent on these effects of the unknown physics at huge energies, then the evaporating black hole scenario could turn out to be reliable after all.

The assumption that long-lived trapping horizons exist in the low-energy physical description (a prediction of standard general relativity) calls for some hypothesis about the characteristics of the high-energy physics. It is difficult, if not impossible, to catalogue all the possible scenarios that might be at work at high energy scales. However, it seems reasonable that they could be separated into two classes: those in which the classical notion of a long-lasting trapping horizon is preserved and those in which it is not. Does the causal unidirectional property of a trapping horizon survive to high-energy physics corrections or is it only a low-energy property? The simplest way to grasp the effects associated with these two possible scenarios is to analyze linear effective quantum field theories containing dispersive corrections of either subluminal or superluminal character, respectively. We cannot logically discard the possibility that the underlying high-energy theories, which either preserve the horizon completely or maintain it only as a low-energy property, do not give rise to the predictions of these sub/superluminal effective field theories. However, we find this possibility difficult to imagine and, in principle, unjustified, as it would contradict the very essence of using effective physical descriptions.

These dispersive analyses were introduced by Jacobson in $[5,6]$ and soon followed by Unruh [7]. At present there are many works analyzing the effects of non-relativistic dispersion relations (see the review [8] and references therein). Typically the conclusion of these investigations can be summarized by saying that Hawking-like emission survives, under mild conditions, the presence of modified dispersion relations of both kinds, and so that the evaporating black-hole scenario is reliable. Sometimes, it is even tacitly assumed that the real (astrophysical) Hawking radiation would be produced in one of these modified manners. However, we want to highlight in this paper that one cannot just study the effect of the modified dispersion relations in Hawking emission without further considerations. Rather, one has to probe the consistency of the whole scenario as well, analyzing collateral effects of dispersion, which may well become central.

In this paper we shall present a comparative discussion of the three different scenarios that one might envisage in the light of the trans-Planckian disaster of the standard evaporating black hole scenario. The stress will be put on how a final consistent scenario might look like. In the first two scenarios it is assumed that a long-lived trapping horizon has been formed from the point of view of the low-energy physical description. The first scenario (section 3) is one with subluminal dispersion relations: the notion of horizon 
remains in the underlying high-energy theory. The second scenario (section 4) involves superluminal dispersion relations: the notion of horizon does not survive in the underlying high-energy theory. Finally, in the third scenario (section 5) we assume from the start that long-lived trapping horizons do not form in the first place. Black holes are substituted by black stars, bodies hovering right outside but extremely close to their gravitational radius. We will show that there exists a mechanism by which these bodies can emit Hawking-like radiation. In this mechanism huge trans-Planckian frequencies play no role whatsoever, so it can be perfectly described with unmodified relativistic dispersion relations.

\section{Preliminaries}

The original Hawking derivation of the thermal emission relies strongly on the dynamical formation of a horizon. It is similar to a process of "cosmological particle production" but modified due to the presence of a horizon. Cosmological particle production is tied up to the dynamics of spacetime: when spacetime stops expanding and becomes stationary, the production also stops. However, in the Hawking process, even though the dynamics is the agent responsible for the particle production, the particle production never ends once a horizon has formed (in the absence of back-reaction), even if the dynamics stops. The infinite redshifting effect of the horizon makes the short movie of events right before the formation of the horizon to be contemplated from the asymptotic region as a never-ending movie. In the asymptotic form of the radiation only the surface gravity of the black hole enters into play, controlling the temperature of the thermal spectrum. However, as explained in the introduction, this huge red-shifting effect is in turn responsible for the trans-Planckian problem.

With modified dispersion relations starting to show up not far from the Planck energy scale, the dynamics of the formation of the horizon turns out to be unable to produce a stationary emission of particles from the horizon. This is so because subluminal and superluminal behaviours of the types described below produce an effective cut-off in the frequencies. But, as is well known, a plain Planckian cut-off $\omega_{\max }$ completely kills Hawking radiation [5, 9]. However, the presence of modified dispersion relations only kills the standard dynamical way in which Hawking radiation appears, but opens up the door to a quite different mechanism of particle production which does not rely on the dynamics but rather on the characteristics of the configuration itself. This new mechanism, known as mode mixing, occurs whenever, for a particular positive energy (a conserved quantity in stationary configurations), there exist modes with both positive and negative norms with respect to the field theory inner product (see for instance 2.2) below).

An elegant way to treat the appearance of dispersion is the covariant formalism developed by Jacobson [10]. Apart from the metric field, there is a unit timelike vector field $u^{a}$ singularizing the specific reference frame in which one has to measure the scale at which the modifications appear. Using for our discussion the simplest case of a scalar field, the field theories we are interested in satisfy the equation

$$
\left[\square+F\left(h^{a b} \nabla_{a} \nabla_{b}\right)\right] \phi=0
$$


where $h^{a b} \equiv g^{a b}+u^{a} u^{b}$. In the space of solutions of the previous equation one can define a conserved inner product as

$$
\left\langle\phi_{1}, \phi_{2}\right\rangle=-i \int \mathrm{d} \Sigma u^{a}\left(\phi_{1}^{*} \stackrel{\leftrightarrow}{\partial_{a}} \phi_{2}\right)
$$

In the reference frame defined by the vector field $u^{a}$ the dispersion relation associated with this equation in the slowly-varying approximation reads

$$
\omega^{2}=k^{2}+F\left(k^{2}\right),
$$

where $k^{2}=h_{a b} k^{a} k^{b}$.

\section{Subluminal dispersion}

We will consider two types of subluminal dispersion. In the first one, $\omega^{2}$ is monotonically growing with $k$ and approaches a constant value for $k \rightarrow \infty$. In the second one, $\omega^{2}$ increases from $k=0$ till it reaches a maximum value and then decreases back to zero at a finite $k$. Two specific functions representative of both types of behaviour are

$$
F\left(k^{2}\right)=-k^{2}+k_{\mathrm{P}}^{2} \tanh \left(k^{2} / k_{\mathrm{P}}^{2}\right), \quad F\left(k^{2}\right)=-k^{4} / k_{\mathrm{P}}^{2},
$$

where $k_{\mathrm{P}}$ is the Planck wavenumber. In particular, throughout our discussion we will for simplicity assume spherical symmetry and use an effectively one-dimensional spacetime with a metric of the form

$$
\mathrm{d} s^{2}=-\left[1-v(x)^{2}\right] \mathrm{d} t^{2}+2 v(x) \mathrm{d} t \mathrm{~d} x+\mathrm{d} x^{2},
$$

where $v$ is the free-fall velocity from infinity, i.e. the flow velocity in the language of acoustic metrics (we have set the speed of light $c=1$ ). In addition we shall choose $u^{a}=\{1, v\}$.

As shown initially by Unruh [7], mode mixing in the surroundings of the horizon produces a stationary spectrum of particles with thermal properties, if $\omega_{c} \gg \kappa_{\mathrm{H}}$, where $\omega_{c}$ is the threshold frequency below which mode mixing takes place. The spectrum is perfectly Planckian up to frequencies of the order of $\omega_{c}$. It should be noted that some subluminal models still exhibit a trans-Planckian problem. The first subluminal dispersion in (3.1) with asymptotically vanishing flow velocity $v(x \rightarrow \infty) \rightarrow 0$ is such an example: finite- $k$ Hawking modes originate via mode conversion outside the horizon from infinite- $k$ modes in the past, see e.g. [11].

Therefore, in general: yes, a subluminal mechanism could be responsible for black hole evaporation while avoiding the trans-Planckian problem. However, this scenario as a whole maintains some other problems of the standard evaporation picture. On the one hand, it is well known that the existence of trapped surfaces entails the formation of singularities in standard general relativity. Any new theory beyond standard general relativity which incorporates a subluminal behaviour would maintain this tendency to form singularities. Thus, in this scenario one would have to face the information-loss problem [12-14]. It is difficult to see how all the information that went to form a black hole could be released in the very last stages of its evaporation. 
On the other hand, the evaporation of a stellar mass black hole would take $10^{56}$ times the current age of the universe. The existence in this scenario of long-lived trapping horizons enormously delays the time at which we will be able to have experimental feedback about the real nature of the underlying high-energy theory. Therefore, in the absence of primordial black holes [15] or microscopic black holes at the LHC [16], ${ }^{1}$ this scenario does not allow an open positive exploration of the full nature of its underlying physics, at least not to the human race as we understand it. As physicists we find this idea, to say the least, disturbing.

\section{Superluminal dispersion}

Superluminal scenarios are completely different. The physics at work inside the horizon becomes exposed to the outside world. In particular, the characteristics of their internal geometry can have an influence on how black holes radiate and more in general on how they behave. The analysis of stationary configurations with a black hole horizon has shown that mode mixing is also able to provide a mechanism for Hawking-like emission in a superluminal scenario (see [17] and [18] for a more recent approach). In those analyses, there is always a crucial assumption regarding how the in-vacuum state looks like near the place where the internal classical singularity is supposed to be. This amounts to selecting a boundary condition at the singularity. One way to have a good control of this boundary condition is to substitute the place where the classical singularity would appear by an internal asymptotic region. Different vacuum states can be selected by allowing or not the net entrance of particles from this internal asymptotic region.

Contrarily to subluminal scenarios, any new theory beyond standard general relativity that incorporates a superluminal behaviour would blur the very essence of the horizon. There will be high-wavenumber signals that can escape from the hole. Actually, assuming that the superluminal correction does not saturate, at any location inside the black hole, there will always be a wavenumber above which the signals can escape towards the exterior. Under these circumstances it is no longer sensible to assume that the theory will have a tendency to form singularities. Quite the opposite view would be more reasonable, namely, that the internal region is perfectly regular so that any wave entering into the hole would after some time travel back towards the horizon. Superluminal dispersion would therefore not only remove the horizon, but also smooth away the curvature singularity.

When in the internal region one allows the existence of particles travelling towards the horizon, that is, when the vacuum state is not the so-called in vacuum, the particle spectrum in the asymptotic region could be largely distorted from a Planckian form (see [19] for a discussion on the various vacuum states one could set the system in). For instance, a similar situation can be seen in the analysis of configurations with a black and a white horizon where a black-hole-laser effect is generated [20-23]. There, the existence of an internal white horizon completely destroys Hawking radiation, giving place instead to a self-amplified creation of particles.

\footnotetext{
${ }^{1}$ We find this possibility much more speculative. In turn, the existence of these microblack holes would be revolutionary, implying that the relevant quantum gravity scale is not the Planck scale but the much smaller electroweak scale.
} 
Moreover, as we are going to show, under reflecting boundary conditions at some point located inside the hole, the theory becomes unstable, as already observed in the context of BECs [24, 25]. This means that there appear frequencies in its spectrum with positive imaginary part (again, very much as in the presence of a combination of a black-hole and a white-hole horizon). To be more specific, let us analyze a scalar quantum field with a quartic superluminal dispersion relation

$$
F\left(k^{2}\right)=k^{4} / k_{\mathrm{P}}^{2}
$$

in a one-dimensional spacetime with a metric (3.2) in which the velocity $v$ has a step-like profile, i.e. such that it vanishes in the exterior and has a constant and homogeneous value $v>1$ in the interior. As before, the spatial dimension represents the radial coordinate of a more realistic spherically symmetric three-dimensional configuration. Furthermore, let us assume that the field is fully reflected at some point inside the hole located at a distance $L$ from the horizon (corresponding to an $s$-wave analysis and a symmetry condition at $r=0$ in three dimensions). Then, from the numerical analysis, we can estimate the number of instabilities $n_{\text {inst }}$, as well as their maximal typical growth rates $\Gamma_{\max }$ and the real part $\omega_{n}$ of their frequencies, as functions of $v$ and the size $L$ of the inner region. The details of this numerical instability analysis can be found in the appendix. Overall, the qualitative behaviour of these quantities is

$$
\begin{aligned}
\Gamma_{\max } & \sim 2(v-1) / L, & n_{\text {inst }} & \sim 2 \omega_{c} / \Gamma_{\max }, \\
\omega_{n} & \sim \pm \Gamma_{\max } n & (n & \left.=1 \ldots n_{\text {inst }} / 2\right),
\end{aligned}
$$

where $\omega_{c}$ is the critical frequency (frequency above which there is no more mode mixing [18]). These results are in agreement with the numerical and analytical studies carried out for the black/white-hole case in ref. [21]. The interpretation of these quantities is straightforward and could actually have been obtained from a qualitative analysis of the problem, as discussed in what follows. $\Gamma_{\max }$ is directly related to the inverse of the time needed to travel back and forth inside the hole. The maximum real part of the frequency of these instabilities is just $\omega_{c}$. These two observations are closely related to the fact that the leaking of resonant modes inside the hole is responsible for the instability. The number of instabilities is determined by the ratio between these two quantities $\omega_{c} / \Gamma_{\max }$, at least for sufficiently large $L$. Again, this is just a consequence of the fact that the resonant modes, which obey a kind of quantization rule, build up and eventually leak through the slightly permeable horizon giving rise to the unstable behaviour.

As an example, figure 1 shows the map of instabilities for a black hole whose interior size is $40 k_{\mathrm{P}}^{-1}$ and the flow velocity is twice the speed of light. In this plot, the growth rate $\operatorname{Im}(\omega)$ is measured in units of $\Gamma_{\max }$ and $\operatorname{Re}(\omega)$ is measured in units of $\omega_{c}$.

If we consider a Solar mass Schwarzschild black hole written in Painlevé-Gullstrand coordinates (in which the radial part of the metric is of the form (3.2)), it is a trivial exercise to show that the average flow velocity in the interior is precisely twice the speed of light. Since its size is typically $3 \mathrm{~km}$, we have the following numbers for the quantities above:

$$
\Gamma_{\max } \sim 2 \cdot 10^{5} \mathrm{~s}^{-1}, \quad n_{\text {inst }} \sim 10^{38}, \quad \omega_{c} \sim 10^{43} \mathrm{~s}^{-1} .
$$




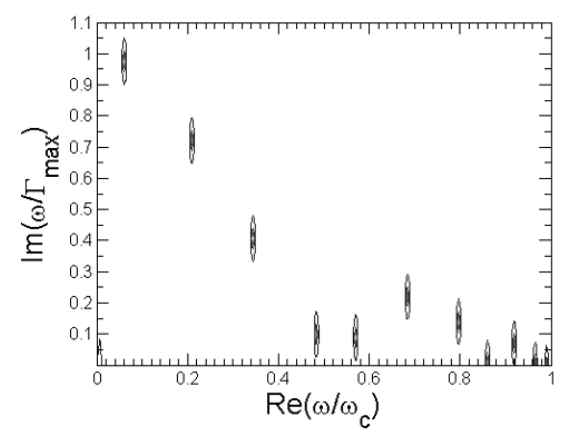

Figure 1. Map of instabilities for a black hole with superluminal dispersion and reflecting boundary conditions, whose interior size is $40 k_{\mathrm{P}}^{-1}$ and with a flow velocity twice the speed of light. The growth rate $\operatorname{Im}(\omega)$ is measured in units of $\Gamma_{\max }$ and $\operatorname{Re}(\omega)$ in units of $\omega_{c}$.

The essential ingredients for this result are the appearance of additional propagation channels characterized by a high-energy scale $\left(k_{\mathrm{P}}\right.$ in (4.1)) and a superluminal character. Our qualitative conclusions are therefore valid beyond the particular model that we have analyzed numerically. Note that although the temporal scale of the instability is very large compared with Planck time and would therefore be seen as metastable from the artificial black-hole perspective, it is actually tiny in astrophysical terms. This means that a Solar mass black hole with superluminal dispersion would be extremely unstable in astrophysical terms and would tend to eliminate its horizon in microseconds. Thus, we see that if the true curvature singularity is smoothed away due to the presence of superluminal dispersion relations (as discussed above), black holes as such would be very unstable. In other words, this analysis suggests that any theory beyond classical general relativity that incorporates superluminal behaviour will avoid the formation of long-lasting trapping horizons.

\section{$5 \quad$ Avoidance of long-lasting horizons}

The last scenario that we will consider is one in which we assume from the start that there are no astrophysical bodies with strict long-lasting trapping horizons. Black holes are substituted by material bodies with a real surface which is hovering right outside, but extremely close, to their gravitational radius. The existence of these objects seems largely implausible within standard general relativity, but they might occur in a modified theory of gravity such as semiclassical general relativity (see the discussion in [26]) or condensed-matter-inspired emergent gravity [27]. Using the terminology of [26] we will generically call them black stars. There are several black-hole mimickers proposed in the literature [26, 28-30]. What we are going to describe here does not depend on the specific reasons behind their existence or on their internal constitution, but only on the fact of staying close to horizon formation.

The entropy associated with these black stars would not have a purely geometrical origin as is typically assumed for black holes. Instead, it will be associated with their matter content under the influence of gravity. It is however remarkable that there exist strong indications that the entropy associated with matter configurations on the verge of forming a 
horizon would follow an area law [31-35]. It is also remarkable that objects of this kind are able to emit radiation by a process closely related to the one originally devised by Hawking. In [36] it was shown that objects without horizons can emit Hawking-like radiation during any period of exponential approach to horizon formation. This observation is resonant with the fact noticed previously in [37] that a collapsing shell bouncing or stopping close to the horizon will emit a burst of thermal radiation.

For instance, imagine a black star vibrating between two radii $r_{\mathrm{H}}+x_{\text {out }}$ and $r_{\mathrm{H}}+x_{\mathrm{in}}$, with both $x_{\mathrm{out}} / r_{\mathrm{H}}, x_{\mathrm{in}} / r_{\mathrm{H}} \ll 1$. To characterize the particle emission associated with a vibration cycle we can use the techniques developed in $[4,38,39]$. Let us consider a simple scalar quantum field over a $1+1$ geometry. The amount of particle production will be characterized by the Bogoliubov coefficients, which can be calculated from the expression [36]

$$
\beta_{\omega \omega^{\prime}}=\frac{1}{2 \pi} \sqrt{\frac{\omega}{\omega^{\prime}}} \int \mathrm{d} u e^{-i \omega^{\prime} U(u)} e^{-i \omega u},
$$

provided that the relation between two different outgoing null coordinates of the geometry $U=U(u)$ is known. Typically, $U$ and $u$ represent asymptotic past and future null coordinates, respectively. Instead of using a complete model for the vibrating geometry, one can alternatively use a stationary Schwarzschild-black-hole geometry and encode the vibrational features into the definition of a non-stationary vacuum state (see [4] for a more detailed discussion). With this aim, let us take a vibrating timelike trajectory $r(\tau)$ connecting $r_{\mathrm{H}}+x_{\text {out }}$ and $r_{\mathrm{H}}+x_{\mathrm{in}}$. An asymptotic observer will label the different null rays by the null parameter

$$
u:=t-r^{*}
$$

where $r^{*}$ is the "tortoise" coordinate

$$
r^{*}:=r+2 m \ln \left(\frac{r}{2 m}-1\right) .
$$

These same null rays can also be labelled in terms of the proper time $\tau$ of the vibrating trajectory. Given a vibrating trajectory we can obtain the relation $u=u(U)$ we are searching for, if we identify $U \equiv \tau$. By using the Schwarzschild metric it is straightforward to see that

$$
u(U)=\int \mathrm{d} U \frac{\left[1+\left(1-\frac{2 m}{r(U)}\right)^{-1} \dot{r}^{2}(U)\right]^{1 / 2}}{\left(1-\frac{2 m}{r(U)}\right)^{1 / 2}}-r^{*}(U) .
$$

Now, given the relation $u=u(U)$ one can calculate the Bogoliubov coefficients. As we are only interested in the presence of radiation with nearly Planckian spectrum, we can instead calculate directly the quantity $[38,39]$

$$
\kappa(u):=-\frac{\mathrm{d}^{2} U}{\mathrm{~d} u^{2}}\left(\frac{\mathrm{d} U}{\mathrm{~d} u}\right)^{-1}=\left.\frac{\mathrm{d}^{2} u}{\mathrm{~d} U^{2}}\left(\frac{\mathrm{d} u}{\mathrm{~d} U}\right)^{-2}\right|_{U(u)} .
$$

This function represents the peeling of geodesics in the geometry and is the magnitude relevant for encoding the radiative properties in situations that go beyond the standard 
scenarios usually analyzed in the context of Hawking radiation, for example, in the presence of dynamical horizons or in the absence of a strict trapping horizon. As explained thoroughly in $[38,39]$, when this function is constant $\kappa(u) \simeq \kappa\left(u_{*}\right)=: \kappa_{*}$ over a sufficiently large interval around a given $u_{*}$, one can ascertain that during this same interval the system is producing a Hawking flux of particles with a temperature

$$
T=\frac{\kappa_{*}}{2 \pi} .
$$

Let us now calculate this quantity for the trajectories of interest. Instead of the variable $r$, let us use $x:=r-2 m$. As we will always be in the regime $x / 2 m \ll 1$, we can simplify (5.4) which becomes $u(U) \simeq u_{\mathrm{a}}(U)$, where

$$
u_{\mathrm{a}}(U)=2 m \int \mathrm{d} U \frac{|\dot{x}|}{x}\left(1+\frac{x}{2 m \dot{x}^{2}}\right)^{1 / 2}-2 m \ln \frac{x}{2 m},
$$

so that

$$
\begin{aligned}
\frac{\mathrm{d} u_{\mathrm{a}}}{\mathrm{d} U}= & 2 m \frac{|\dot{x}|}{x}\left(1+\frac{x}{2 m \dot{x}^{2}}\right)^{1 / 2}-2 m \frac{\dot{x}}{x}, \\
\frac{\mathrm{d}^{2} u_{\mathrm{a}}}{\mathrm{d} U^{2}}= & -2 m \operatorname{sign}(\dot{x})\left(\frac{\dot{x}}{x}\right)^{2} \frac{1+\frac{x}{4 m \dot{x}^{2}}-\frac{x \ddot{x}}{\dot{x}^{2}}}{\left(1+\frac{x}{2 m \dot{x}^{2}}\right)^{1 / 2}} \\
& +2 m\left(\frac{\dot{x}}{x}\right)^{2}\left(1-\frac{x \ddot{x}}{\dot{x}^{2}}\right) \\
\frac{\mathrm{d}^{2} u_{\mathrm{a}}}{\mathrm{d} U^{2}}\left(\frac{\mathrm{d} u_{\mathrm{a}}}{\mathrm{d} U}\right)^{-2}= & -\operatorname{sign}(\dot{x}) \frac{\frac{1}{4 m}+\ddot{x}}{\left(1+\frac{x}{2 m \dot{x}^{2}}\right)^{1 / 2}-\ddot{x} .}
\end{aligned}
$$

Now, consider a trajectory $x(\tau)$ such that it is in free-fall from $x_{\text {out }}$ to $x_{\text {in }}$ where it bounces back symmetrically to return to $x_{\text {out }}$ (and so on and so forth). Except at the bounce itself the acceleration of the trajectory is $\ddot{x} \simeq-1 / 4 m$, i.e., it is equal to the surface gravity acceleration of the black hole. Then, if one plugs this value of the acceleration in (5.9), it is easy to realize that

$$
\kappa(u) \simeq \frac{\mathrm{d}^{2} u_{\mathrm{a}}}{\mathrm{d} U^{2}}\left(\frac{\mathrm{d} u_{\mathrm{a}}}{\mathrm{d} U}\right)^{-2} \simeq \frac{1}{4 m} .
$$

For instance, figure 2 shows a free-fall trajectory with a regularized bounce and figures 3 and 4 , the resulting behaviour for $\kappa(u)$. Therefore, a black star continuously vibrating in this way will continuously emit a Hawking-like flux of particles with a Planckian spectrum, only contaminated by a small non-Planckian emission associated with the bounces.

Even if the vibration did not have these precise characteristics, one would still find a time interval within each cycle for which $\kappa(u) \simeq$ constant and hence an overall approximately constant $\kappa$ as long as the vibration cycle does not depart significantly from a free-fall trajectory. As seen from infinity the duration of a vibration half-cycle can be estimated to be

$$
\Delta t_{v} \simeq 4 m \ln \frac{x_{\mathrm{out}}}{x_{\mathrm{in}}}
$$




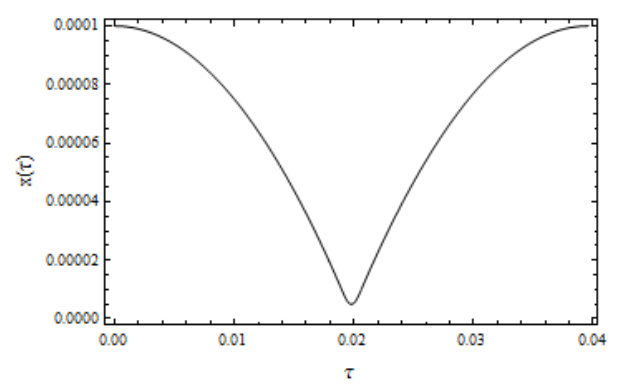

Figure 2. Depiction of the trajectory associated with a single vibration of a black star object. The trajectory is free-fall between $x_{\text {out }}=0.0001$ and $x_{b}=0.00001$, then it connects with an elastic bounce between $x_{\mathrm{b}}$ and $x_{\mathrm{in}}=0.000005$. Finally, it goes back to $x_{\text {out }}$ in free-fall, before starting a new vibration cycle. We use $2 m=1$ units.

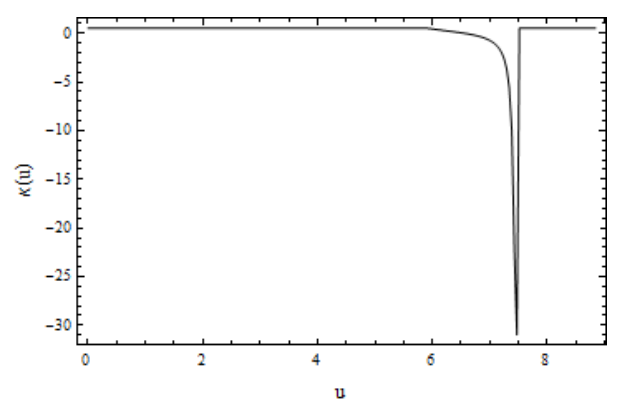

Figure 3. Exact value of $\kappa(u)$ for the vibration of figure 2 in $2 m=1$ units.

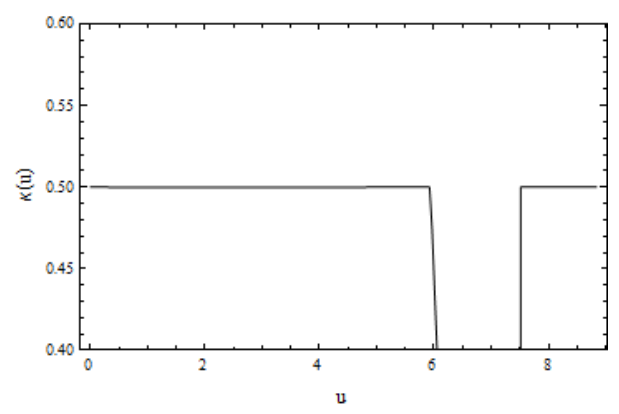

Figure 4. Exact value of $\kappa(u)$ for the vibration of figure 2 in $2 m=1$ units (detail). The approximate $\kappa \simeq(4 m)^{-1}=0.5$ value is seen to be almost exact in the free-fall regions.

With a sufficiently large ratio $x_{\text {out }} / x_{\text {in }}$ this duration can be made several times larger than $\kappa_{\mathrm{H}}^{-1}=4 m$, the inverse of the peak frequency of the Hawking spectrum. There would then be two options depending on the time resolution of the particle detector at infinity. With low temporal resolution we will see a Planckian spectrum with an additional peak at the vibration frequency, while with higher temporal resolution (but still low enough to see the Hawking spectrum), we will observe a Hawking spectrum with short periodic bursts of 
additional (non-thermal) radiation due to the bounces.

In the case of the black stars of ref. [26], objects always on the verge of falling into their own gravitational radius, this would happen not only for the body as a whole, but also for each ball of matter contained within every internal radius. The pulsations of such a black star would thus resemble standard stellar pulsations in the sense of depending on the internal structure. Notice that, overall, the energy density profile of the black star would follow an almost $1 /\left(4 \pi r^{2}\right)$ law. The closer the radius to the centre of the star, the bigger the surface gravity associated with this radius. Thus a vibration of an internal part of the black star will produce black body radiation with a larger temperature than the one associated with the surface gravity of the real external surface of the star. However, this radiation will be more red-shifted on its way out towards infinity. These two effects exactly compensate so that any radiation at infinity will be seen with the same temperature no matter at which precise radius it originated. The in principle non-coherent superposition of the radiation coming from vibrations at different radii would smooth out possible fluctuations on the value of $\kappa$ due to the actual vibration profiles. The final situation would be something practically indistinguishable from the perfectly continuous (non-fluctuating) emission deduced by Hawking.

In view of this discussion, it is not unreasonable that the generalized laws of black hole thermodynamics based on the geometrical notion of horizon area serve as a good approximation to the much "dirtier" physics of these material objects, which could have an area-entropy law and a Hawking emission at the appropriate temperature.

Regarding the central theme of this paper, the point that we want to highlight is that the maximum frequency involved in the calculation of the particle production associated with these vibrations or bounces is not unreasonably large (remember that in this section we are assuming a relativistic dispersion relation). The maximum frequency invoked in one vibration will be of the order

$$
\omega_{\max } \sim \kappa_{\mathrm{H}} e^{\kappa_{\mathrm{H}} \Delta t_{v}} .
$$

For a solar mass black hole and even for an oscillation with a semi-period as large as $\Delta t_{v} \sim$ $100 / \kappa_{\mathrm{H}}$, which corresponds to a ratio $x_{\text {out }} / x_{\text {in }} \sim 10^{43}$, this cut-off value $\omega_{\max } \sim 10^{4} \omega_{\mathrm{P}}$ is perfectly compatible with the stringent bounds on Lorentz symmetry breaking imposed by current observations [40, 41]. To have a better idea of the behaviour of this magnitude, for an oscillation between $x_{\mathrm{in}} \simeq 10^{-20} r_{\mathrm{H}}$ and $x_{\text {out }} \simeq 10^{-10} r_{\mathrm{H}}$, we have $\kappa_{\mathrm{H}} \Delta t_{v} \simeq 23$, so that $\omega_{\max } \sim 10^{-29} \omega_{\mathrm{P}}$. When the system develops a new vibration, the frequencies involved in the process do not blue-shift further but are once again below the previous maximum frequency. There is then no trans-Planckian problem of any sort in this scenario. The maximum energy-scale for which we have to assume that a relativistic quantum field theory is reliable is perfectly reasonable. Therefore, within this scenario the prediction that there will be Hawking-like emission is absolutely reliable. Moreover, as we have shown, this radiation is Planckian in shape, but it is not thermal, in the sense that there aren't any hidden correlations to trace over. The partners of the Hawking particles are not trapped behind a horizon, so that they are also emitted towards infinity if only with a time delay. In the absence of a horizon, as well as of a singularity, the information-loss problem is completely absent in this scenario. 


\section{Summary and conclusions}

The mechanism for thermal emission of a black hole found by Hawking contains a transPlanckian problem that ultimately makes it extremely unreliable. Using this very problem as a guiding principle, we have presented and discussed three different scenarios, with three different Hawking-like emission mechanisms, in which the trans-Planckian problem does not show up. The trans-Planckian problem is caused by the combined effect of having a long-lived trapping horizon and a relativistic field theory at work up to huge energy scales. Thus, the three scenarios that we have analyzed are:

1. Assume a long-lived horizon but effective modifications of the dispersion relations of subluminal type;

2. Assume a long-lived horizon but effective modifications of the dispersion relations of superluminal type;

3. Keep a relativistic dispersion relation, but eliminate from the start the presence of strict long-lived trapping horizons.

In the first scenario the existence of a long-lived trapping horizon at the classical level of general relativity (a unidirectional barrier for signalling) is preserved by the high-energy physics of the underlying theory. Therefore, this scenario suffers from the informationloss problem of the standard evaporation scenario and makes it impossible in practice to empirically explore the physics at work inside the horizon.

In the second scenario the existence of a long-lived trapping horizon is just an effective description at low energies. The high-energy physics of the underlying theory allows for superluminal signalling and so the interior of the hole is exposed to the outside world. However, as we have shown, this scenario is not self-consistent in an astrophysical context. Under the reasonable assumption that superluminality would regularize the internal singularity, configurations with a horizon become extremely unstable in astrophysical terms. Once formed, a Solar-mass black hole would tend to eliminate its horizon in a time scale of tens of microseconds. The apparently stationary astrophysical bodies standardly regarded as black holes should then be something different, without any horizons whatsoever.

This leads us to the third scenario, in which we assume from the start that the astrophysical bodies usually called black holes are instead black stars, material objects hovering right outside but extremely close to their gravitational radius. We have shown that vibrations of these objects can result in a Hawking-like emission. The maximum mode frequencies involved in this emission mechanism are very much under control and never reach large trans-Planckian values. Therefore, for the effective description of this emission mechanism, it is not necessary to consider any deviations from the relativistic dispersion relations.

The emission of these objects will be Planckian although not strictly thermal. Since there aren't any horizons, there will be no hidden correlations and no information-loss problem: The Hawking partners will be emitted if only with a short time delay. On top of Hawking-like emission the spectrum will also show some short periodic pulsations associated with the bounces in each vibration cycle. This could eventually serve as an experimen- 
tal signature of these objects. An effort to explore whether there exists a compelling dynamical mechanism behind the existence of these speculative black-star objects is on-going.

\section{Acknowledgments}

The authors want to thank Renaud Parentani, Stefano Finazzi, Stefano Liberati, Iacopo Carusotto, Grigory Volovik and Matt Visser for illuminating discussions. Financial support was provided by the Spanish MICINN through the projects FIS2008-06078-C03-01 and FIS2008-06078-C03-03 and by the Junta de Andalucía through the project FQM219. G.J. is supported by a FECYT postdoctoral mobility contract of the Spanish MEC/MICINN, and also acknowledges the Academy of Finland (Centers of Excellence Programme 20062011, grant 218211) and the EU 7th Framework Programme (FP7/2007-2013, grant 228464 Microkelvin).

\section{A Stability analysis}

In this appendix, we provide the details of the numerical analysis carried out to find the instabilities of a scalar field that obeys the modified two-dimensional Klein-Gordon equation

$$
\left(\partial_{t}+\partial_{x} v\right)\left(\partial_{t}+v \partial_{x}\right) \phi=\left(\partial_{x}^{2}-\frac{1}{k_{\mathrm{P}}^{2}} \partial_{x}^{4}\right) \phi .
$$

The dispersion relation associated with this equation is

$$
(\omega-k v)^{2}=k^{2}\left(1+k^{2} / k_{\mathrm{P}}^{2}\right) .
$$

This dispersion relation is also found in BECs [24, 42]; in fact, the analysis below is similar to that of instabilities and quasi-normal modes in BECs [25, 43], except for using different boundary conditions. In particular, we will analyze flow velocities $v$ with step-like profiles, i.e. such that they vanish in the exterior and have a constant and homogeneous value $|v|>1$ in the interior. By convention, the flow will be directed towards the left so that $-1<v=v_{r}<0$ in the outer region $x>0$ while $v=v_{l}<-1$ in the inner region $x<0$. Furthermore, let us assume that the field is fully reflected at some point inside the hole located at a distance $L$ from the horizon, i.e. at $x=-L$.

The matching conditions at the point $x=0$ where the velocity profile is discontinuous can be obtained by requiring that the third spatial derivative $\phi^{\prime \prime \prime}$ of the field should also be discontinuous there and compensate for the jump in $v$ in the generalized Klein-Gordon equation. This translates into the four conditions

$$
[\phi]=\left[\phi^{\prime}\right]=\left[\phi^{\prime \prime}\right]=0, \quad\left[v \partial_{t} \phi+v^{2} \phi^{\prime}+\phi^{\prime \prime \prime} / k_{\mathrm{P}}^{2}\right]=0,
$$

where $[f]:=f(\epsilon)-f(-\epsilon)$.

For a given frequency $\omega$, the general solution can be written as

$$
\phi= \begin{cases}\sum_{j=1}^{4} A_{j} e^{i\left(k_{j} x-\omega t\right)} & (x<0), \\ \sum_{j=5}^{8} A_{j} e^{i\left(k_{j} x-\omega t\right)} & (x>0),\end{cases}
$$


where $\left\{k_{j}\right\}$ are the roots of the corresponding dispersion equations (four roots for each homogeneous region), and the constants $A_{j}$ have to be such that the matching conditions (A.3) are satisfied. We can write down these conditions in matrix form $\sum_{j} \Lambda_{i j}(\omega) A_{j}=0$, where the transpose of $\Lambda$ is

$$
\Lambda_{i j}^{T}=\left(\begin{array}{rrrc}
1 & k_{1} & k_{1}^{2} & v_{l} \omega-v_{l}^{2} k_{1}+k_{1}^{3} / k_{\mathrm{P}}^{2} \\
1 & k_{2} & k_{2}^{2} & v_{l} \omega-v_{l}^{2} k_{2}+k_{2}^{3} / k_{\mathrm{P}}^{2} \\
1 & k_{3} & k_{3}^{2} & v_{l} \omega-v_{l}^{2} k_{3}+k_{3}^{3} / k_{\mathrm{P}}^{2} \\
1 & k_{4} & k_{4}^{2} & v_{l} \omega-v_{l}^{2} k_{4}+k_{4}^{3} / k_{\mathrm{P}}^{2} \\
-1 & -k_{5} & -k_{5}^{2} & -\left(v_{r} \omega-v_{r}^{2} k_{5}+k_{5}^{3} / k_{\mathrm{P}}^{2}\right) \\
-1 & -k_{6} & -k_{6}^{2} & -\left(v_{r} \omega-v_{r}^{2} k_{6}+k_{6}^{3} / k_{\mathrm{P}}^{2}\right) \\
-1 & -k_{7} & -k_{7}^{2} & -\left(v_{r} \omega-v_{r}^{2} k_{7}+k_{7}^{3} / k_{\mathrm{P}}^{2}\right) \\
-1 & -k_{8} & -k_{8}^{2} & -\left(v_{r} \omega-v_{r}^{2} k_{8}+k_{8}^{3} / k_{\mathrm{P}}^{2}\right)
\end{array}\right) .
$$

Furthermore, to find the unstable modes these conditions have to be complemented with conditions at the boundaries of the system, namely at $x \rightarrow \infty$ and $x=-L$. We will deal only with instabilities so we will be concerned with modes with $\operatorname{Im}(\omega)>0$. Furthermore, for each mode with frequency $\omega$, there is another mode with frequency $-\omega^{*}$. So we will consider only $\operatorname{Re}(\omega)>0$ without loss of generality.

For complex $\omega$ the four roots of the dispersion relation in the right region are complex: Two of them with positive imaginary part (say $k_{5}$ and $k_{6}$ ) and two of them with negative imaginary part (say $k_{7}$ and $k_{8}$ ) [24]. Since the modes must be normalizable, we must ensure that

$$
A_{7}=A_{8}=0,
$$

so that the solution does not grow exponential at $x \rightarrow \infty$. No additional requirements (e.g. outgoing conditions) are imposed at infinity so the boundary conditions we use match the analysis in [18, 24, 44].

Let us now analyze the reflection at $x=-L$. In order to find appropriate boundary conditions, we have to notice that in the presence of dispersion, the current

$$
J_{x}=\phi^{*} \overleftrightarrow{\partial_{x}} \phi-\frac{1}{k_{\mathrm{P}}^{2}} \phi^{*} \overleftrightarrow{\partial_{x}^{3}} \phi
$$

is locally conserved, so that it should vanish at $x=-L$. One can choose different reflecting boundary conditions (generalization of the Dirichlet, Neumann and Robin boundary conditions). Qualitatively our results do not depend on this choice. For concreteness we have used generalized Dirichlet conditions:

$$
\phi(-L)=0, \quad \phi^{\prime}(-L)=0,
$$

which amount to the following conditions on the modes:

$$
\sum_{j=1}^{4} A_{j} e^{-i k_{j} L}=0, \quad \sum_{j=1}^{4} A_{j} k_{j} e^{-i k_{j} L}=0 .
$$


These four conditions (A.6) and (A.9) can be imposed by adding the following extra rows to the $4 \times 8$ matrix $\Lambda(\omega)$ :

$$
\left(\begin{array}{cccccccc}
0 & 0 & 0 & 0 & 0 & 0 & 1 & 0 \\
0 & 0 & 0 & 0 & 0 & 0 & 0 & 1 \\
e^{-i k_{1} L} & e^{-i k_{2} L} & e^{-i k_{3} L} & e^{-i k_{4} L} & 0 & 0 & 0 & 0 \\
k_{1} e^{-i k_{1} L} & k_{2} e^{-i k_{2} L} & k_{3} e^{-i k_{3} L} & k_{4} e^{-i k_{4} L} & 0 & 0 & 0 & 0
\end{array}\right) .
$$

The extended $8 \times 8$ matrix $\tilde{\Lambda}(\omega)$ thus obtained is such that the possible unstable modes must satisfy $\sum_{j} \tilde{\Lambda}_{i j}(\omega) A_{j}=0$. This set of equations will have a solution for every value of $\omega$ at which its determinant vanishes.

The numerical method then follows these steps:

1. Given the values of the flow velocity in the left $v_{l}$ and the right $v_{r}$, for each frequency $\omega$ in a grid covering the first quadrant of the complex plane, we calculate its associated $k$-roots [by solving the dispersion relation (A.2)] in both regions.

2. Given the value of the size $L$ of the interior of the black hole, for each value of $\omega$, we evaluate the $\operatorname{determinant} \operatorname{det}[\tilde{\Lambda}(\omega)]$. The values of $\omega$ for which this determinant vanishes correspond to instabilities of the system.

3. Instead of drawing contour plots of $\operatorname{det}[\tilde{\Lambda}(\omega)]$, in order to increase contrast, we plot the following function:

$$
F(\omega)=-\log [f(\omega)] / \max \{-\log [f(\omega)]\},
$$

where

$$
\begin{aligned}
f(\omega) & =g(\omega) / \max [g(\omega)], \\
g(\omega) & =\operatorname{det}[\tilde{\Lambda}(\omega)] /\langle[\operatorname{det} \tilde{\Lambda}(\omega)]\rangle,
\end{aligned}
$$

and $\langle h\rangle$ stands for a coarse graining of the function $h$ that removes the peaks, hence leaving only the background behaviour of $h$. The maxima of this function $F(\omega)$ correspond to vanishing values of $\operatorname{det}(\tilde{\Lambda})$ and hence to instabilities of the system, as in figure 1 above.

\section{References}

[1] S. Hawking, Black hole explosions, Nature 248 (1974) 30 [INSPIRE].

[2] S. Hawking, Particle creation by black holes, Commun. Math. Phys. 43 (1975) 199 [Erratum ibid. 46 (1976) 206] [INSPIRE].

[3] W.G. Unruh, Notes on black hole evaporation, Phys. Rev. D 14 (1976) 870 [InSPIRE].

[4] L.C. Barbado, C. Barceló and L.J. Garay, Hawking radiation as perceived by different observers, Class. Quant. Grav. 28 (2011) 125021 [arXiv:1101.4382] [INSPIRE].

[5] T. Jacobson, Black hole evaporation and ultrashort distances, Phys. Rev. D 44 (1991) 1731 [INSPIRE]. 
[6] T. Jacobson, Black hole radiation in the presence of a short distance cutoff, Phys. Rev. D 48 (1993) 728 [hep-th/9303103] [INSPIRE].

[7] W.G. Unruh, Sonic analog of black holes and the effects of high frequencies on black hole evaporation, Phys. Rev. D 51 (1995) 2827 [INSPIRE].

[8] C. Barceló, S. Liberati and M. Visser, Analogue gravity, Living Rev. Rel. 14 (2011) 3 [gr-qc/0505065] [INSPIRE].

[9] C. Barceló, L. Garay and G. Jannes, Sensitivity of Hawking radiation to superluminal dispersion relations, Phys. Rev. D 79 (2009) 024016 [arXiv: 0807.4147] [INSPIRE].

[10] T. Jacobson and D. Mattingly, Gravity with a dynamical preferred frame, Phys. Rev. D 64 (2001) 024028 [gr-qc/0007031] [INSPIRE].

[11] T. Jacobson, Trans Planckian redshifts and the substance of the space-time river, Prog. Theor. Phys. Suppl. 136 (1999) 1 [hep-th/0001085] [INSPIRE].

[12] S. Hawking, Breakdown of predictability in gravitational collapse, Phys. Rev. D 14 (1976) 2460 [INSPIRE].

[13] S. Hawking, Information loss in black holes, Phys. Rev. D 72 (2005) 084013 [hep-th/0507171] [INSPIRE].

[14] J. Preskill, Do black holes destroy information?, presented at International symposium on black holes, membranes, wormholes and superstrings, Woodlands U.S.A. January 1992 [hep-th/9209058] [INSPIRE].

[15] B. Carr, K. Kohri, Y. Sendouda and J. Yokoyama, New cosmological constraints on primordial black holes, Phys. Rev. D 81 (2010) 104019 [arXiv:0912.5297] [INSPIRE].

[16] CMS collaboration, V. Khachatryan et al., Search for microscopic black hole signatures at the Large Hadron Collider, Phys. Lett. B 697 (2011) 434 [arXiv: 1012.3375] [INSPIRE].

[17] S. Corley, Computing the spectrum of black hole radiation in the presence of high frequency dispersion: an analytical approach, Phys. Rev. D 57 (1998) 6280 [hep-th/9710075] [INSPIRE].

[18] J. Macher and R. Parentani, Black/white hole radiation from dispersive theories, Phys. Rev. D 79 (2009) 124008 [arXiv:0903.2224] [INSPIRE].

[19] C. Barceló, L. Garay and G. Jannes, The two faces of quantum sound, Phys. Rev. D 82 (2010) 044042 [arXiv: 1006.0181] [INSPIRE].

[20] S. Corley and T. Jacobson, Black hole lasers, Phys. Rev. D 59 (1999) 124011 [hep-th/9806203] [INSPIRE].

[21] S. Finazzi and R. Parentani, Black-hole lasers in Bose-Einstein condensates, New J. Phys. 12 (2010) 095015 [arXiv: 1005.4024] [INSPIRE].

[22] A. Coutant and R. Parentani, Black hole lasers, a mode analysis, Phys. Rev. D 81 (2010) 084042 [arXiv:0912.2755] [INSPIRE].

[23] U. Leonhardt and T.G. Philbin, Black hole lasers revisited, arXiv:0803.0669 [INSPIRE].

[24] L. Garay, J. Anglin, J. Cirac and P. Zoller, Sonic black holes in dilute Bose-Einstein condensates, Phys. Rev. A 63 (2001) 023611 [gr-qc/0005131] [INSPIRE].

[25] C. Barceló, A. Cano, L. Garay and G. Jannes, Stability analysis of sonic horizons in Bose-Einstein condensates, Phys. Rev. D 74 (2006) 024008 [gr-qc/0603089] [InSPIRE]. 
[26] C. Barceló, S. Liberati, S. Sonego and M. Visser, Fate of gravitational collapse in semiclassical gravity, Phys. Rev. D 77 (2008) 044032 [arXiv:0712.1130] [INSPIRE].

[27] C. Barceló, L. Garay and G. Jannes, Quantum non-gravity and stellar collapse, Found. Phys. 41 (2011) 1532 [arXiv:1002.4651] [INSPIRE].

[28] P.O. Mazur and E. Mottola, Gravitational condensate stars: an alternative to black holes, gr-qc/0109035 [INSPIRE].

[29] P.O. Mazur and E. Mottola, Gravitational vacuum condensate stars, Proc. Nat. Acad. Sci. 101 (2004) 9545 [gr-qc/0407075] [inSPIRE].

[30] G. Chapline, E. Hohlfeld, R. Laughlin and D. Santiago, Quantum phase transitions and the breakdown of classical general relativity, Int. J. Mod. Phys. A 18 (2003) 3587 [gr-qc/0012094] [INSPIRE].

[31] R.D. Sorkin, R.M. Wald and Z.J. Zhang, Entropy of selfgravitating radiation, Gen. Rel. Grav. 13 (1981) 1127 [INSPIRE].

[32] F. Pretorius, D. Vollick and W. Israel, An operational approach to black hole entropy, Phys. Rev. D 57 (1998) 6311 [gr-qc/9712085] [INSPIRE].

[33] G. Abreu and M. Visser, Tolman mass, generalized surface gravity and entropy bounds, Phys. Rev. Lett. 105 (2010) 041302 [arXiv:1005.1132] [INSPIRE].

[34] G. Abreu, C. Barceló and M. Visser, Entropy bounds in terms of the w parameter, arXiv: 1109.2710 [INSPIRE].

[35] J.P. Lemos and O.B. Zaslavskii, Entropy of quasiblack holes, Phys. Rev. D 81 (2010) 064012 [arXiv:0904.1741] [INSPIRE].

[36] C. Barceló, S. Liberati, S. Sonego and M. Visser, Hawking-like radiation does not require a trapped region, Phys. Rev. Lett. 97 (2006) 171301 [gr-qc/0607008] [INSPIRE].

[37] C.R. Stephens, G. 't Hooft and B.F. Whiting, Black hole evaporation without information loss, Class. Quant. Grav. 11 (1994) 621 [gr-qc/9310006] [INSPIRE].

[38] C. Barceló, S. Liberati, S. Sonego and M. Visser, Minimal conditions for the existence of a Hawking-like flux, Phys. Rev. D 83 (2011) 041501 [arXiv:1011.5593] [InSPIRE].

[39] C. Barceló, S. Liberati, S. Sonego and M. Visser, Hawking-like radiation from evolving black holes and compact horizonless objects, JHEP 02 (2011) 003 [arXiv:1011.5911] [INSPIRE].

[40] T. Jacobson, S. Liberati and D. Mattingly, Lorentz violation at high energy: concepts, phenomena and astrophysical constraints, Annals Phys. 321 (2006) 150 [astro-ph/0505267] [INSPIRE].

[41] L. Maccione, A.M. Taylor, D.M. Mattingly and S. Liberati, Planck-scale Lorentz violation constrained by ultra-high-energy cosmic rays, JCAP 04 (2009) 022 [arXiv:0902.1756] [INSPIRE].

[42] L. Garay, J. Anglin, J. Cirac and P. Zoller, Black holes in Bose-Einstein condensates, Phys. Rev. Lett. 85 (2000) 4643 [gr-qc/0002015] [INSPIRE].

[43] C. Barceló, A. Cano, L. Garay and G. Jannes, Quasi-normal mode analysis in BEC acoustic black holes, Phys. Rev. D 75 (2007) 084024 [gr-qc/0701173] [INSPIRE].

[44] S. Finazzi and R. Parentani, Spectral properties of acoustic black hole radiation: broadening the horizon, Phys. Rev. D 83 (2011) 084010 [arXiv:1012.1556] [INSPIRE]. 